\title{
Thymoma B2 without Myasthenia Gravis or Any Autoimmune Disorder-A Case Report
}

\author{
Ajith DJ*1, Mashhadi M1, Wesley $\mathrm{H}^{2}$ and Peter S1 \\ ${ }^{1}$ Senior Research Scientist, GMC Cardiothoracic and Vascular Research Society, India \\ ${ }^{2}$ Research Officer, GMC Cardiothoracic and Vascular Research Society, India
}

*Corresponding Author: Deva Japa Ajith MD, Department of Pathology, Senior

\section{Case Report}

Volume 4 Issue 2

Received Date: June 26, 2019

Published Date: July 18, 2019

Research Scientist, GMC Cardiothoracic and Vascular Research Society, DDMM Heart Institute, Mission Road, Nadiad387002, India, Email: devajapa@ddmmheart.org

\section{Abstract}

The association of Thymoma with Myasthenia Gravis (MG) is well known. Thymoma without MG or any auto immune disease is however, very rare. Since these tumors arise from thymocytes which are immature T lymphocytes of different maturation which has antigen expression profile. Thymoma B1 and B2 are more commonly associated with MG and immunehistochemical analysis play a vital role in differentiating these thymoma types. Our case showed positivity for PANCK, CD57 and negativity for SMA, Desmin, Vimentin, HMB45, EMA, CK7, S100 and CD5. We present an interesting case of Thymoma B2 without pre-operative MG and no evidence of MG for five years of follow up.

Keywords: Immunohistochemistry; Thymocytes

\section{Clinical Summary}

A 50-year-old male patient with a history of left sided chest pain since one month was admitted to the hospital without any symptoms of MG like muscular weakness mainly fatigability of skeletal muscles. There was no other notable medical history; in particular, there was no evidence of red cell aplasia or hypo gamma globulinaemia. Examination of the cardiovascular system was normal and respiratory system showed bilateral air entry. AntiAChR antibodies were not detected and electromyogram was not performed. On further evaluation, CT chest scan showed a left anterior mediastinal mass -possibility of a Germ cell tumor. Post-surgical diagnosis confirmed the thymoma by histopathology as well as by Immunohistochemistry.

\section{Pathological findings}

We received a globular encapsulated mass (Figure1) measuring $8.5 \times 7.5 \times 4.5 \mathrm{~cm}$ in which cut section showed multiple nodules separated by septate. The nodules didn't show the evidence of cystic appearance or necrosis.

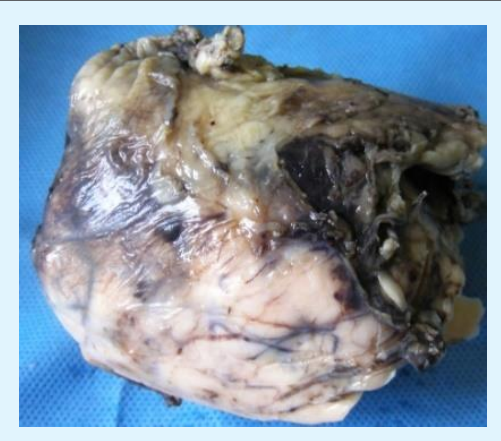

Figure 1: Encapsulated tumor of the thymus. 


\section{Anaesthesia \& Critical Care Medicine Journal}

\section{Histopathology}

Histologically the lesion showed a mixture of neoplastic epithelial cells and nonneoplastic lymphocytes (Figure2) which were predominant and the cells were arranged in lobules. The cells which are round to spindle shaped with oval vesicular nuclei and conspicuous nucleoli. There is evidence of perivascular palisading with elongated cells. Immunohistochemical analysis was done for the differentiation of thymomas types. Epithelial cells showed positivity for PAN CK (Figure3), mature lymphocytes were positive for LCA, CD57 (Figure 4) and negativity was seen in CK7 and Pan-T cell marker CD5.

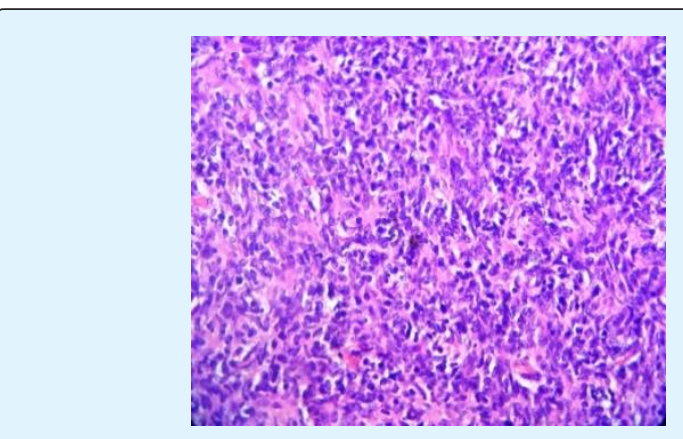

Figure 2: Mixture of neoplastic epithelial cells and lymphocytes (Hematoxylin and Eosin stain 10x view).

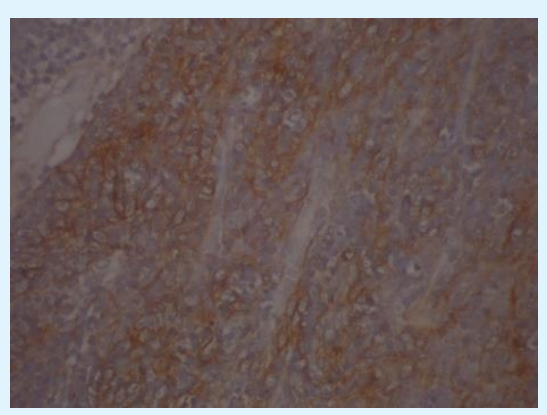

Figure 3: PAN CK - Positive for thymic epithelial cells.

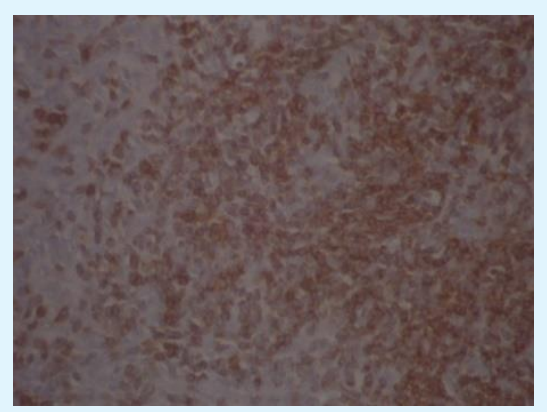

Figure 4: CD 57 - Positive for mature lymphocytes (1).

\section{Discussion}

Thymoma refers to a malignancy arising from thymocytes which are epithelial cells of the thymus [1]. Among the anterior mediastinum primary neoplasms, thymomas are the commonest [2]. Thymoma is a rare tumor which has a prevalence of 0.1 to $0.4 / 100,000$ and the proportion of B2 types accounts for about $20 \%$ for all thymomas [3]. Thymoma is predominantly seen in the fourth or fifth decade of life and there is no gender predilection [4]. Thymomas are classified according to the dominant cell type as epithelial, lymphocytic, mixed, or spindle-cell type [4]. In 1999, a World Health Organization (WHO) labeled as Type A, B, B1, B2, B3 and for the heterogeneous group of thymic carcinomas, collectively called Type $C$ thymomas [3]. The difference between $\mathrm{B} 1$ and $\mathrm{B} 2$ depends on the size of the epithelial cells will be "larger and more numerous", and with no areas of "medullary" differentiation in type B2 compared with type B1 [4]. Thymomas are diagnosed incidentally while imaging because they reflect no symptom due to its site while half of the patients presents with symptoms due to intrathoracic spread such as superior vena cava obstruction, or with symptoms of an associated paraneoplastic disorder [5]. Thymomas are associated with immune, non-immune, and endocrine disorders and the most common associations are with Myasthenia Gravis (MG), Pure Red cell Aplasia (PRCA),and hypogammaglobulinemia [6]. All patients evaluated for signs and symptoms of myasthenia gravis who are suspected of having thymoma and should be treated prior to surgery to avoid complications during anesthesia [7]. Type B1 and B2 tumors are commonly associated with MGwhile type $A$ and $A B$ tumors will not reveal the rarity of $M G$ [5]. It is plausible that in thymoma tumor associated patients may have symptoms such as hypogammaglobulinaemia, hypothermia which results from autoantibody directed against a variety of selfantigens induce hypothalamic injury and altered thermoregulation [7].

Thymomas exhibit a unique biology and are associated with a number of paraneoplastic disorders such as myasthenia gravis, red cell aplasia, or hypogamma globulinaemia. Immunohistochemically, the neoplastic epithelial component of the thymomas expresses keratin as well as express epithelial membrane antigen [8]. Approximately $30 \%-40 \%$ of thymomas are invasive. The malignant nature of this tumor depends on the invasion either macroscopically into surrounding organs and structures or microscopically through the thymus capsule [5]. The WHO histologic subtype is an independent 


\section{Anaesthesia \& Critical Care Medicine Journal}

prognostic factor in Stage I and II thymomas, among which WHO Type A, AB, and B1 thymomas form a lowrisk group. Given the heterogeneity of WHO type B thymomas, Masaoka stage should always be considered when predicting prognosis and planning treatment, including adjuvant treatment for patients with type $\mathrm{B}$ thymomas [9].

Clinically thymomas were classified by the Masaoka Staging. Stage 1 is macroscopically encapsulated and no capsular invasion microscopically. Our case represents stage1. In general, thymoma cannot be differentiated grossly from the less common thymic epithelial malignancies, thymic carcinoma and thymic carcinoid, but both thymic carcinoma and carcinoid are more aggressive and typically present with local and/or metastatic spread [10]. Type $B$ tumors are more invasive than type $A$ and $A B$ tumors [7]. Immunohistochemical analysis is useful in assisting in the identification of these neoplasms. Epithelial cells of thymomas show Keratin positivity while EMA negative. Lymphocytes of Immature $\mathrm{T}$ phenotype shows positive with CD1a, CD99, TdT and T cells express most pan-T cell markers CD3, CD5 [11].

\section{Conclusion}

In conclusion, because thymomas possess potential for unique biology for paraneoplastic syndromes and local recurrence, a multidisciplinary approach is recommended for the evaluation and treatment of these tumors.The prognosis among subtypes within type B thymomas according to both Masaoka staging system and the WHO classification system shows that the status of capsular integrity in such cases will be a more accurate predictor of their biological potential and future behaviour than the histology. In our case, self-tolerance and the expression of auto immune regulator may be the key factors for not contributing to any autoimmune disease preoperative and post-surgery for duration of five years.

\section{References}

1. Chahinian AP (2003) Thymomas and Thymic Tumors.
2. Okumura $M$, Ohta $M$, Tomiyama $N$, Minami $M$, Hirabayashi $\mathrm{H}$, et al. (2002) WHO classification in thymoma. Kyobu Geka 55(11): 916-920.

3. Dadmanesh F, Sekihara T, Rosai J (2001) Histologic typing of thymoma according to the new World Health Organization classification. Chest Surg Clin N Am 11(2): 407-420.

4. Bernatz PE, Harrison EG, Clagett OT (1961) Thymoma: a clinicopathologic study. J Thorac Cardiovasc Surg 42: 424-444.

5. Riedel RF, Burfeind WR (2006) Thymoma: Benign Appearance, Malignant Potential. The Oncologist 11(8): 887-894.

6. Johns RH, Reinhardt AK (2009) Association between thymoma and persistent hypothermia: a case report. J Med Case Reports 3: 73.

7. Suster S (2006) Diagnosis of thymoma. J Clin Pathol 59(12): 1238-1244.

8. Kim HK, Choi YS, Kim J, Shim YM, Han J, et al. (2010) Type B thymoma: is prognosis predicted only by World Health Organization classification? J Thorac Cardiovasc Surg 139(6): 1431-1435.

9. Kondo K, Yoshizawa K, Tsuyuguchi M, Kimura S, Sumitomo M, et al. (2004) WHO histologic classification is a prognostic indicator in thymoma. Ann Thorac Surg 77(4): 1183-1188.

10. Marom EM (2013) Advances in thymoma imaging. J Thorac Imaging 28(2): 69-80.

11. Li S, Juco J, Mann KP, Holden JT(2004) Flow cytometry in the differential diagnosis of lymphocyterich thymoma from precursor T-cell acute lymphoblastic leukemia/lymphoblastic lymphoma. Am J Clin Pathol 121(2): 268-274. 